\title{
FINITE ELEMENT SIMULATION OF 4D-PRINTED SMA-SMP COMPOSITE ACTUATORS
}

\author{
AMIR HOSEIN SAKHAEI ${ }^{1}$, SAEED AKBARI ${ }^{2}$ AND QI GE ${ }^{3}$ \\ ${ }^{1}$ School of Engineering and Digital Arts, University of Kent, \\ Canterbury, CT2 7NT, United Kingdom \\ a.sakhaei@kent.ac.uk \\ ${ }^{2}$ RISE Research Institutes of Sweden \\ Box 104, SE-431 22 Mölndal, Sweden \\ saeed.akbari@ri.se \\ ${ }^{3}$ Department of Mechanical and Energy Engineering, Southern University of Science and Technology \\ Shenzhen 518055, China \\ geq@sustech.edu.cn
}

Key words: 4D printing, SMA-SMP Composite Actuator, Finite Element Modelling.

\begin{abstract}
The shape memory alloy-shape memory polymer (SMA-SMP) composite actuators are the novel smart actuator designs which capable of large recoverable actuation deformation as well as retaining shape in the actuated state without consuming extra energy. This is particularly doable because of the recent progresses in additive manufacturing technologies. The 4D printed actuators designed in the previous study [7] are fabricated by multi-material inkjet 3D printing and is able to deposit various range of digital materials with different elastic stiffness in micro-scale. In this study, we have developed the numerical simulation of the 4D-printed composite actuator hinges to determine important actuation parameters and develop a design tool for future applications. The three-dimensional finite element models were conducted in ABAQUS and the nonlinear temperature dependence behaviour of shape memory alloy (SMA) and shape memory polymer (SMP) materials are incorporated through the user material subroutines (UMATs). The finite element results then have been compared with the experimental measurements and illustrate the accuracy of our numerical analysis. Therefore, the validated computational framework could be used for further development of actuators that could generate complex deformations.
\end{abstract}

\section{INTRODUCTION}

Over the last decades, researchers were designing different types of actuators using smart materials $[1,2,3,4]$. These types of smart materials can generate the displacement after receiving the appropriate stimulus. For example, shape memory alloys (SMAs) as one of the most famous smart materials could retain their original shape after being trained by mechanical or thermal loads [5]. However, SMAs alone is not enough to fabricate the large deformation actuators as the recoverable strain is very small in SMAs $(4-8 \%)[5,6]$. 
Therefore, researchers proposed soft polymeric composites with embedded SMA wires in it. The location of SMA wires in the soft polymeric matrix can generate large actuation in the form of bending, twisting or a combination of both $[2,7,8]$.

Over the last years, there were different approaches to improve the performance of SMAelastomer composite actuators and make them a suitable design for various applications. One approach which is related to the topic of this paper is using shape memory polymers (SMP) as matrix or part of the matrix in the structure of composite and fabricate SMA-SMP actuator composites $[7,9,10]$. The rationale behind this approach is to improve the performance of the actuator in shape retainment and recovery. This means SMA-SMP actuator composites can retain their actuated (deformed) shape after deactivation of stimulation source such as electrical voltage. This is significantly important as this type of shape retainment could decrease the energy consumption, cost of operation and unnecessary heat generation. Furthermore, the implemented SMP in the composite can accelerate and modify the recovery step which is another benefit for this type of actuators [7].

The fabrication of SMA-SMP composites accelerated after recent developments in additive manufacturing technologies which allow engineers and researchers to create SMP components with detailed geometry and as a part of a composite without the limitations of moulding process. Furthermore, many of the commercial 3D printers such as multi-material inkjet 3D printers (which are used to fabricate the experimental samples in this study) could deposit various so-called digital materials with broad range of stiffness in the structures. This enables a factor of controlling the properties of SMP in the composite and therefore controlling the performa

Thus, the recent progress

fabricate various type
numerical tool that ena

process. The proposed computati
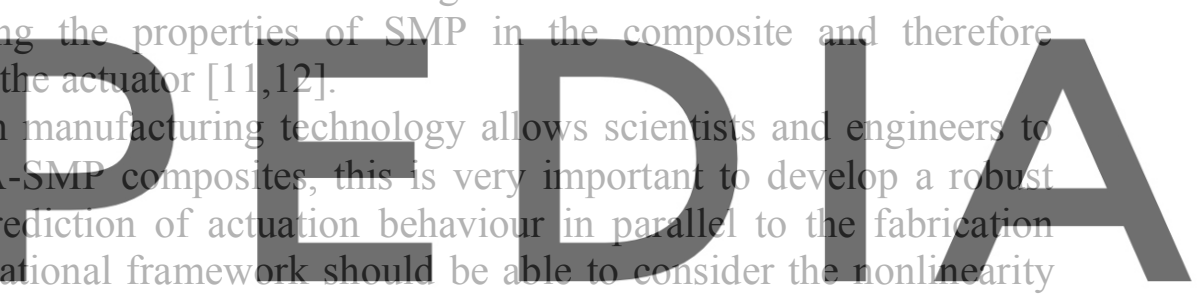

of SMA and SMP mechanical behaviours as well as capacity to tailor the stiffness of the

Register forffred. at https//www.scipedia.com to download the version without the watermark

Therefore, in this study we have presented a three-dimensional finite element framework for 4D-printed SMA-SMP actuator hinges to determine important actuation parameters including shape retainment and recovery and to be used for the design, improvement and analysis process. The three-dimensional finite element models were conducted in ABAQUS [13] and the nonlinear temperature dependence behaviour of SMA and SMP are incorporated through the user material subroutines (UMATs). Furthermore, the numerical model is then validated by comparing the results with experimental results which are developed and performed by the authors. These validations illustrated the accuracy of the numerical analysis and how this could be used for further development of actuators and generation of complex deformations.

The structure of the current paper is as follow: in Section 2, the design of SMA-SMP composite actuator is presented. In section 3, finite element models and condition of simulations are discussed. In Section 4, The results and the discussions are presented and finally, concluding remarks are given in Section 5. 


\section{SMA-SMP COMPOSITE ACTUATOR DESIGN}

The design of the SMA-SMP actuator is presented comprehensively in [7]. A sample design as illustrated in Figure 1 includes two hinges with embedded SMP layer as well as a portion of soft elastomeric segment. The pre-strained (5\%) SMA wires are also implemented parallel to longitudinal axis but with some distance from the neutral axis. SMA wires can be heated by Joule heating when they are connected to a voltage and by contraction after heating, they generate the large bending deformation. Furthermore, the resistive wires are embedded inside the SMP segment to generate Joule heating in this layer. This sample design is capable of large bending deformation when the SMP is in soft rubbery state $\left(T>T_{g}\right)$ and can retain its deformed shape when $T<T_{g}$.

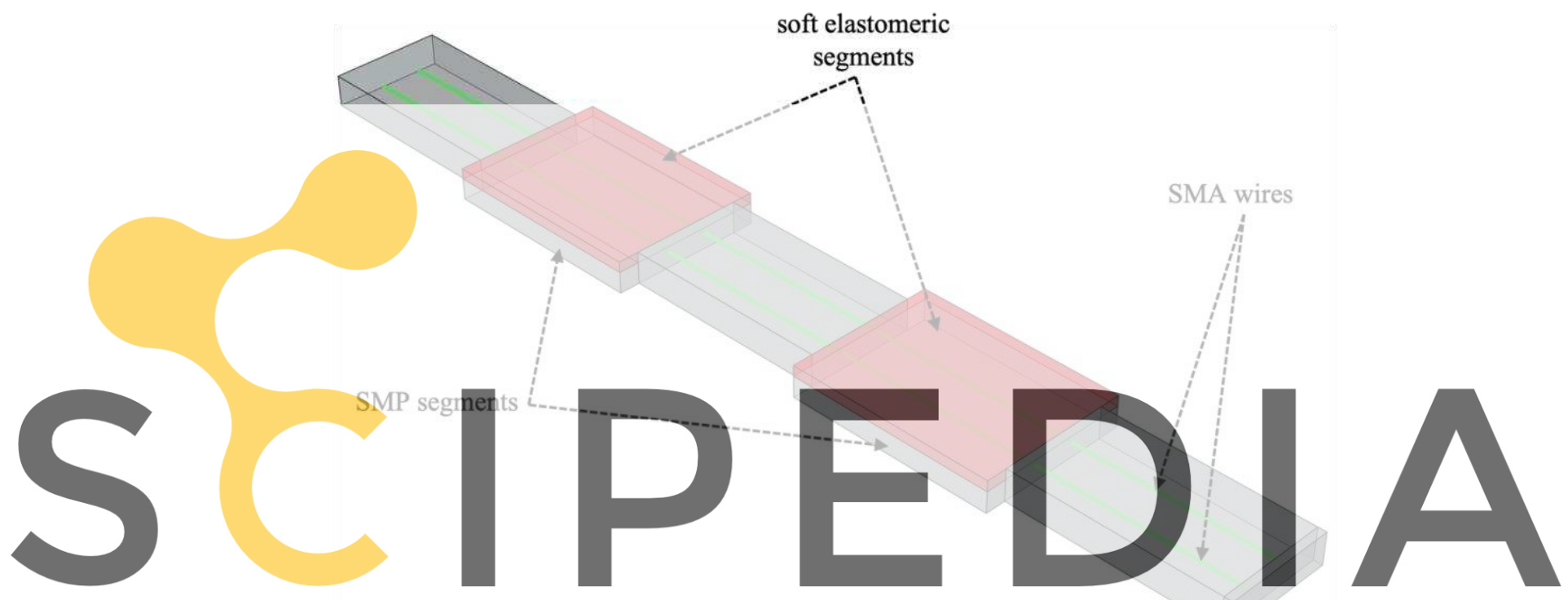

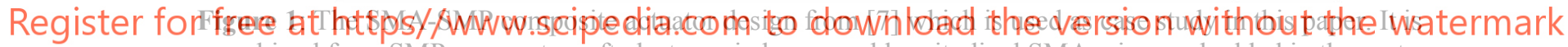
combined from SMP segments, soft elastomeric layers and longitudinal SMA wires embedded in the part.

\section{NUMERICAL SIMULATION OF SMA-SMP COMPOSITE}

We have used ABAQUS software as a commercial finite element tool to develop the threedimensional numerical simulations for the above-mentioned SMA-SMP composite actuator and study the nonlinear thermo-mechanical performance of the composite actuator. The simulations would be then verified by comparing computational results with experimental measurements.

\subsection{Materials modelling}

There are three different type of materials used in the SMA-SMP composite design: (i) shape memory alloy, (ii) shape memory polymer, and (iii) elastomeric material. In the finite element simulations, we should have the correct material model for each of these materials. The materials used here are characterized experimentally in other studies [2,12] therefore, in this paper we only describe the implementation of these behaviours in ABAQUS.

As explained earlier, the multi-material inkjet 3D printer Objet 500 Connex 3, Stratasys 
has been used to fabricate the composite actuator. For the elastomeric part the 3D printer could fabricate different options from so-called Agilus30 with elastic modulus $E \sim 0.4 M P a$ to so-called FLX9995 with elastic modulus $E \sim 14.7 \mathrm{MPa}$. In our experimental sample and our finite element model in this study we have used digital material FLX9960 with linear elastic modulus of 2.6 $\mathrm{MPa}$.

For modelling the behaviour of shape memory alloy material in our simulations, we have implemented the isotropic thermo-mechanical constitutive model developed by $[14,15]$ as an ABAQUS user-defined material (UMAT). According to [14], the Helmholtz free energy per unit reference volume, $\psi$, is given as

$$
\psi=\hat{\psi}\left(\mathbf{E}^{e}, \theta, \xi\right)=\psi^{e}+\psi^{\xi}+\psi^{\theta},
$$

were

$$
\psi^{e}=\widehat{\psi^{e}}\left(\mathbf{E}^{e}, \theta\right)=\frac{1}{2} \mathbf{E}^{e} \cdot \mathbb{C}\left[\mathbf{E}^{e}\right]-\mathbf{A}\left(\theta-\theta_{0}\right) \cdot \mathbb{C}\left[\mathbf{E}^{e}\right],
$$

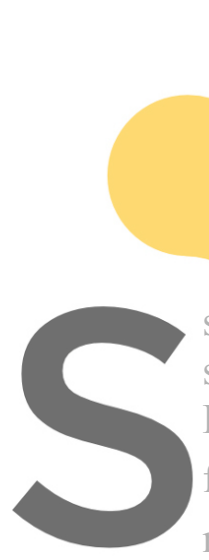

$$
\psi^{\xi}=\widehat{\psi \xi}(\theta, \xi)=\frac{\lambda_{T}}{\theta_{T}}\left(\theta-\theta_{T}\right) \xi
$$

$$
\psi^{\theta}=\widehat{\psi^{\theta}}(\theta) .
$$

Here, $\psi^{e}$ represents the thermo-elastic free energy density, $\mathbb{E}^{e}=\frac{1}{2}\left(\mathbb{C}^{e}-\mathbb{I}\right)$ is the elastic strain, $\mathbb{C}=2 \mu \mathbb{I}+(\kappa-2 \mu / 3) \mathbf{I} \otimes \mathbf{I}$ is the fourth-order elastic moduli tensor and $\mathbf{A}=\alpha \mathbf{I}$ is the
second order thermal expansion tensor with $\alpha$ being the thermal expansion coefficient.
Furthermore, $\lambda_{T}$ is the constant latent heat of phase transformation, $\xi$ s martenstic volume
fraction $(0 \leq \xi \leq 1)$, and $\theta_{T}=\frac{M_{S}+A_{S}}{2}$ is the transformation temperature where $M_{S}$ is the
martensite tart temperature and $A_{S}$ is the austenite start temparature.Knowing the Helmholtz free energy, the stress-strain constitutive law would be

Register for free at https//www.scipedia. $\frac{60 \mathrm{~m}}{\partial \mathrm{E} e}=\mathrm{t}[\mathrm{E}$ dowwhload the version without the wajyermark

As we explained eariier, $\mathbf{E}^{e}=\frac{1}{2}\left(\mathbf{C}^{e}-\mathbf{1}\right)$ is the is the elastic strain and $\mathbf{C}^{e}=\mathbf{F}^{e^{T}} \mathbf{F}^{e}$, where $\mathbf{F}^{e}$ is the elastic distortion and is part of the total deformation gradient $\mathbf{F}=\mathbf{F}^{e} \mathbf{F}^{\text {inel }}$. Therefore, to determine $\mathbf{F}^{e}$ we should first calculate the $\mathbf{F}^{\text {inel }}$ which is the inelastic transformation distortion could be found from

$$
\dot{\mathbf{F}}^{\text {inel }}=\left\{\sqrt{3 / 2} \bar{\varepsilon}_{t} \dot{\xi} \mathbf{S}\right\} \mathbf{F}^{\text {inel }},
$$

where, $\mathbf{S}=\frac{\operatorname{sym}\left(\mathbf{C}^{e} \mathbf{T}\right)_{d e v}}{\left|\operatorname{sym}\left(\mathbf{C}^{e} \mathbf{T}\right)_{d e v}\right|}$ is the inelastic flow direction and $\bar{\varepsilon}_{t}$ is the maximum transformation strain which is a material constant. This material model then implemented as a user-defined material UMAT. The comprehensive details about this constitutive model can be find in [14].

In this study, the SMA wires are from Nickle-Titanium alloy (commercial name Flexinol) with Martensite start temperature $M_{s}=52^{\circ} \mathrm{C}$, Martensite finish temperature $M_{f}=42^{\circ} \mathrm{C}$, Austenite start temperature $A_{s}=68^{\circ} \mathrm{C}$, and Austenite finish temperature $A_{f}=78^{\circ} \mathrm{C}$. Furthermore, the Young's modulus of alloy when it is in martensitic phase is $28 \mathrm{GPa}$ and $75 \mathrm{GPa}$ when it is in the austenitic phase and the maximum value of phase transformation 
strain (recovery strain) of this SMA material which is stated as $\bar{\varepsilon}_{t}$ in our model is $5 \%$.

Finally, to model the behaviour of shape memory polymer, we follow up the multi-branch visco-elastic constitutive model developed by [16,17] and implemented it as another userdefined material (UMAT) for our finite element simulations. SMPs can recover a large deformation by increasing the temperature to above the glass transition temperature, $T_{g}$ therefore, this temperature and the stiffness of the SMP material in the rubbery and glass state has a significant effect on the shape recovery behaviour. The model presented by [16] is a classical spring-dashpot model and consists of an equilibrium and several non-equilibrium branches. Thus, the total Cauchy stress for the SMP material point is presented as

$$
\boldsymbol{\sigma}=\boldsymbol{\sigma}_{e q}+\boldsymbol{\sigma}_{g}+\sum_{i=1}^{m} \boldsymbol{\sigma}_{r}^{i}
$$

where, $\boldsymbol{\sigma}_{g}$ and $\boldsymbol{\sigma}_{r}^{i}$ are the Cauchy stress for nonequilibrium glassy and rubbery branches and $\boldsymbol{\sigma}_{e q}$ is the Cauchy stress in the equilibrium branch and represent the hyperelastic behaviour of material above the $T_{g}$. According to Arruda-Boyce model [18]:

$$
\sigma_{\text {eq }}=\frac{n k_{B} T}{3 J_{M}} \frac{\sqrt{N}}{\lambda_{\text {chain }}} \mathcal{L}^{-1}\left(\frac{\lambda_{\text {chain }}}{\sqrt{N}}\right) \overline{\mathbf{B}}^{\prime}+K\left(J_{M}-1\right) \mathbf{I},
$$

where, $k_{B}$ is the Boltzmann's constant, $n$ is the crosslinking density, $T$ is the temperature, $N$ is the number of Kuhn segments, $K$ is the Bulk modulus, $J_{M}=\operatorname{det} \mathbf{F}_{M}$ and $\mathbf{F}_{M}$ is the deformation gradient representing the mechanical deformation. Furthermore, $\overline{\mathrm{B}}^{\prime}=\overline{\mathrm{B}}-$

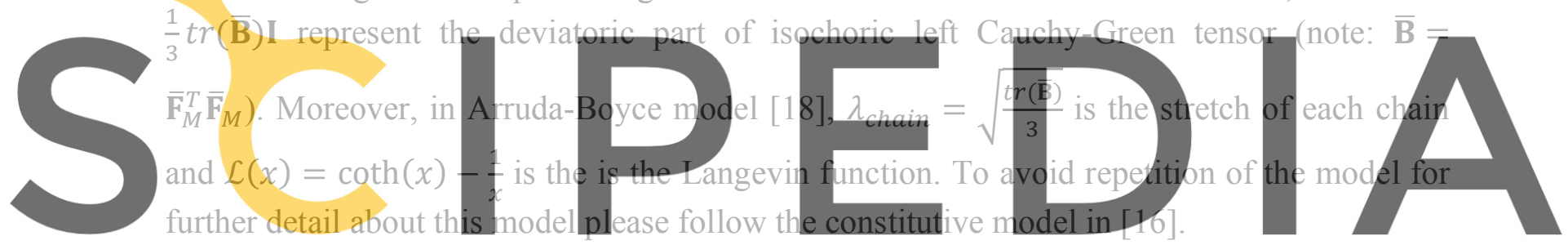

As stated earlier, we have used the multi-material inkjet 3D printer (Objet 500 Connex 3,

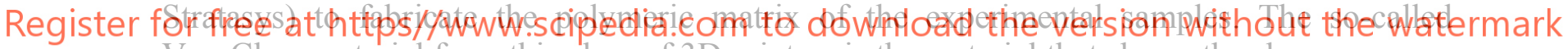
VeroClear material from this class of 3D printers is the material that shows the shape memory behaviour and thus is chosen for fabrication of SMP segments in the experimental samples. The mechanical behaviour of VeroClear is characterised carefully in other studies [2,19] and from there the stiffness of VeroClear changes from $1387 \mathrm{MPa}$ at room temperature where VeroClear is in glassy state to $16 \mathrm{MPA}$ at around $60{ }^{\circ} \mathrm{C}$ which is higher than the glass transition temperature $\left(T_{g}=58{ }^{\circ} \mathrm{C}\right)$ and therefore, the SMP is in rubbery state.

\subsection{Finite Element Model}

The three-dimensional numerical simulations have been constructed in the commercial finite element software, ABAQUS [13], to analyse the thermo-mechanical behaviour of the SMA-SMP composite actuators. As illustrated in Figure 2, due to the symmetrical design of the actuator composite with respect to XY plane, the half of the design is modelled, and the symmetrical boundary condition is implemented on the centreline surface. Figure 2 also represents the mechanical boundary conditions which is fixing the composite at one end while the other end is free to move.

The SMP region in the composite, is modelled using 3568 continuum linear hexahedral 
elements with hybrid formulation so-called C3D8H in ABAQUS, and the SMA wire is implemented by 109 continuum linear line elements, so-called B31H. Furthermore, the soft elastomeric regions fabricated from digital material FLX9960 is modelled by 560 linear hexahedral elements with hybrid formulation so-called C3D8H in ABAQUS and are shown by red in the Figure 2. Finally, the contact between the SMA wire and the inner wall of the hole composite is modelled by a frictionless surface-to-surface model in ABAQUS.

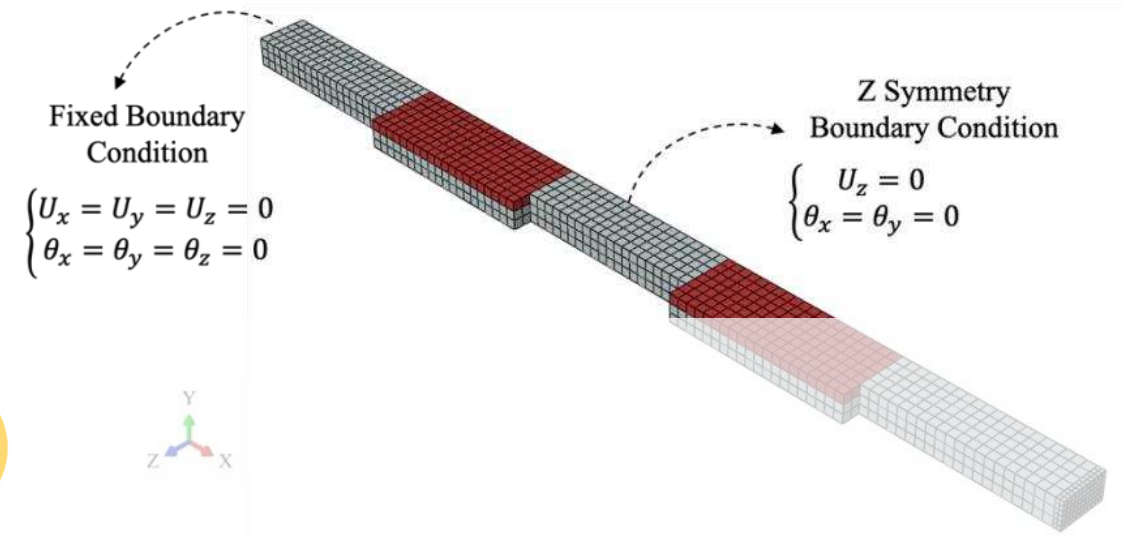

Figure 2: 3D finite element model of the SMA-SMP composite actuator. Due to symmetric design over XY plane, only half of the model is simulated by considering the symmetric boundary condition.
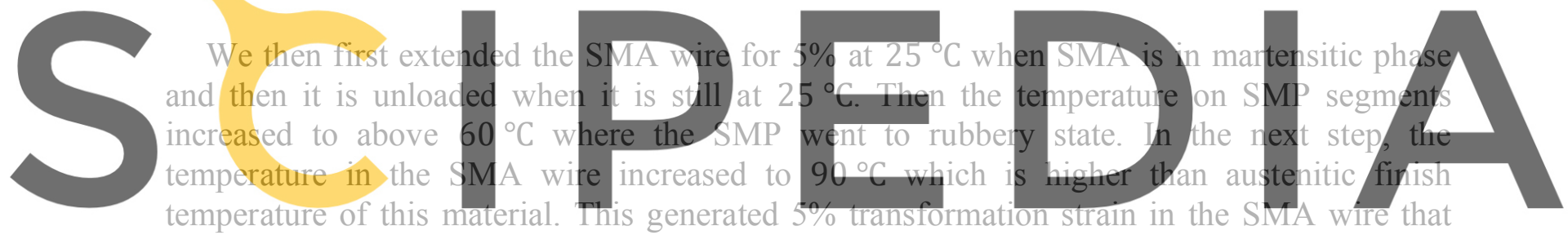

temperature of this material. This generated $5 \%$ transformation strain in the SMA wire that

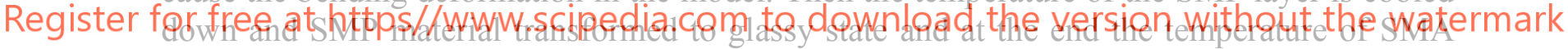

wire is reduced back to $25^{\circ} \mathrm{C}$. Finally, to observe the recovery performance of this composite

actuator, we oniy shouid increase the temperature of SIVIP segments to $60^{\circ} \mathrm{C}$ (above $T_{g}$ ) which activate the shape recovery behaviour of SMP.

\section{RESULTS AND DISCUSSIONS}

In this section, the thermo-mechanical behaviour of SMA-SMP composite actuators is measured and presented by finite element simulations. Then simulation results are compared to the experimental data which have done by the authors in another study [7]. Figure 3 illustrate the simulation result of the activation and recovery of the SMA-SMP actuator design which is presented in Figure 1 earlier. Figures 3-a-c and Figures 3-d-f shows the deformation and recovery of actuator when only SMP in hinge 1 and 2 are activated, respectively. 


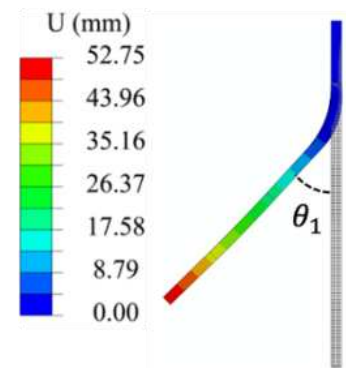

(a)

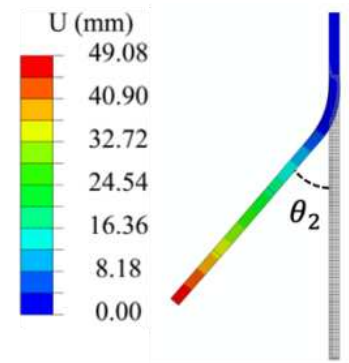

(b)

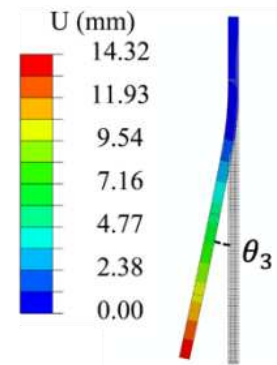

(c)
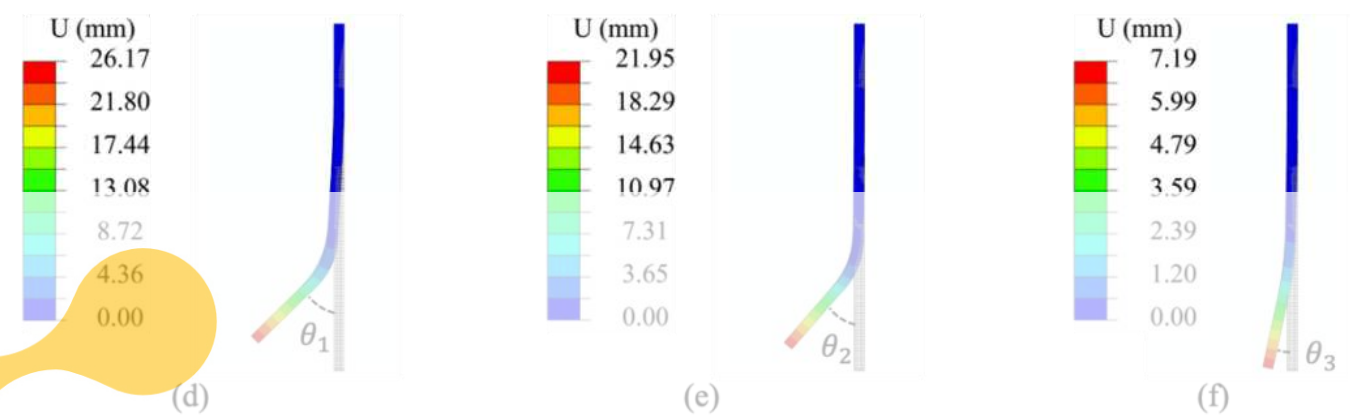

(e)

(f)

Figure 3: Simulation results of the activation of SMP in: (a-c) hinge-1, and (d-f) hinge-2. (a) and (d) are showing

the step after activation of SMP when the SMA wire is still at high temperature. (b) and (e) are showing the
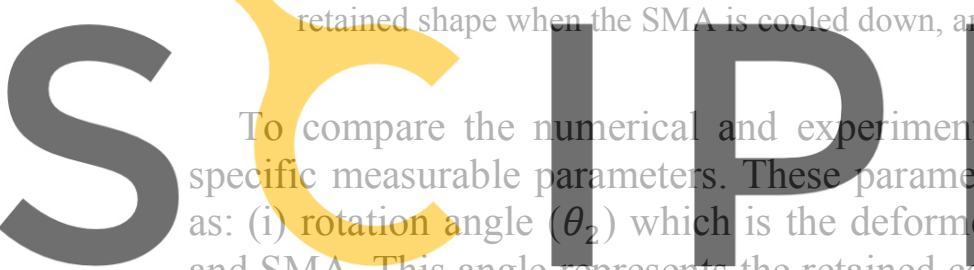

and SMA. This angle

Shape fixity ratio $\left(R_{f}\right)$ which represents the ability to retain the actuated shape after cooling

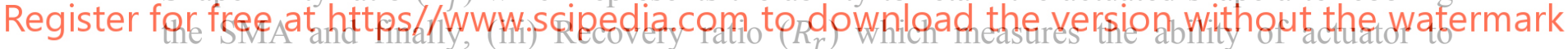

recover to its original shape. Shape fixity ratio and recovery ratio would be measured from

$$
R_{f}=\frac{\theta_{2}}{\theta_{1}}
$$

and

$$
R_{r}=1-\frac{\theta_{3}}{\theta_{2}},
$$

where, $\theta_{1}$ is the maximum bending angle of the actuator when the SMA wire is still at the high temperature (austenite phase), $\theta_{2}$ shows the bending angle after cooling the SMA wire, $\theta_{3}$ is the bending angle after recovery. These angles are illustrated in Figures 9-a to 9-c.

To validate our numerical simulations and to illustrate the potential of the proposed finite element model in predicting the behaviour of the SMA-SMP actuator, we have designed multiple simulations by changing the thickness of SMP layer in the model and measuring the deformed angle $\left(\theta_{2}\right)$, shape fixity ratio $\left(R_{f}\right)$, and shape recovery ratio $\left(R_{r}\right)$. The results then compared to the experimental data.

Figure 4 shows the variation of rotation angle $\left(\theta_{2}\right)$ when the thickness of SMP layer is changing from $0.5 \mathrm{~mm}$ to $3 \mathrm{~mm}$ in the SMA-SMP compote actuator design. Furthermore, the 
comparison between the finite element simulations and experiments also shows the accuracy of the simulations which could be used for further design process.

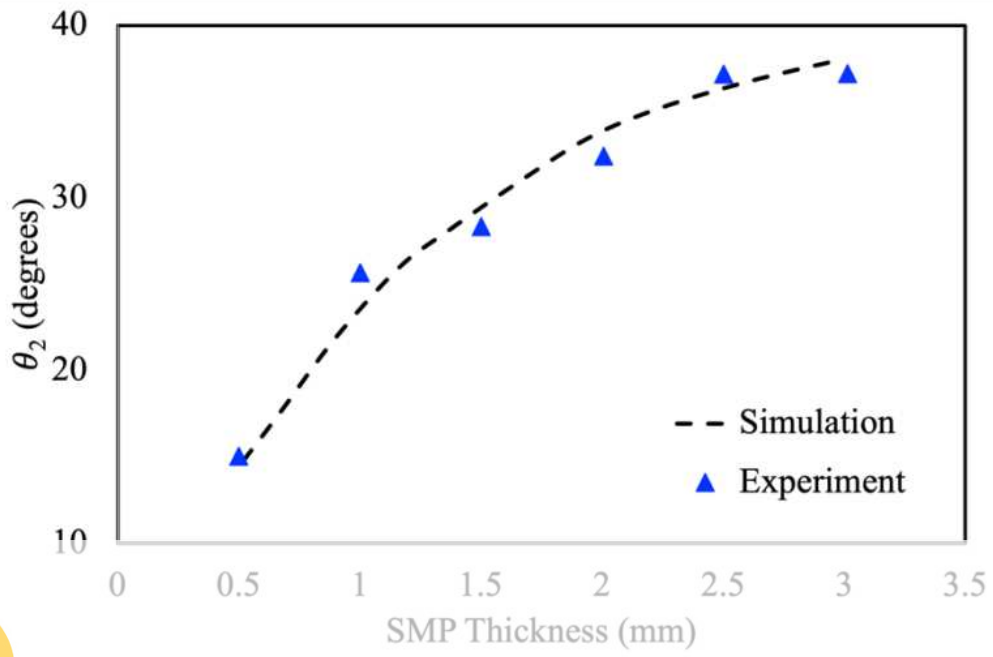

Figure 4: Variation of rotation angle $\left(\theta_{2}\right)$ when the thickness of SMP layer is changing from $0.5 \mathrm{~mm}$ to $3 \mathrm{~mm}$.

The comparison between the computational and experimental measurements of shape fixity ratio $\left(R_{f}\right)$, and shape recovery ratio $\left(R_{r}\right)$ of the actuator design when the thickness of the

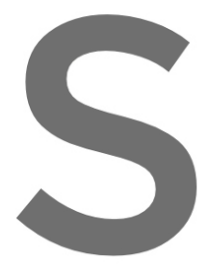
SMP layer is changing respectively. As can be the thickness of the S follow the same trend model.
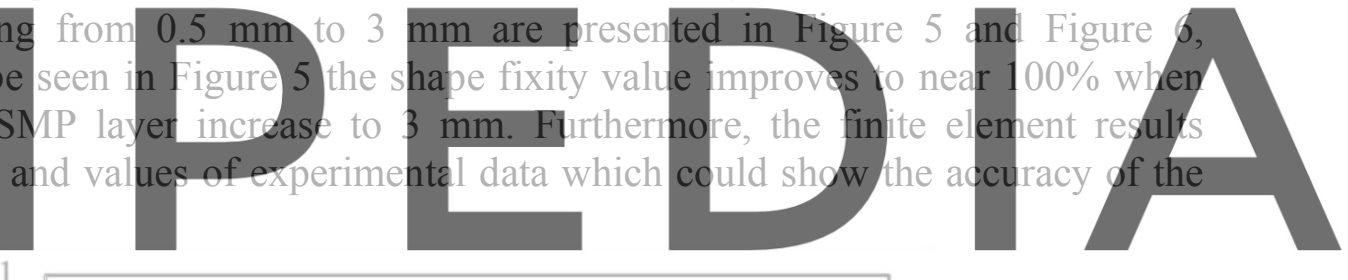

Register for free at https//ywww.scipedia.com to downtlóa

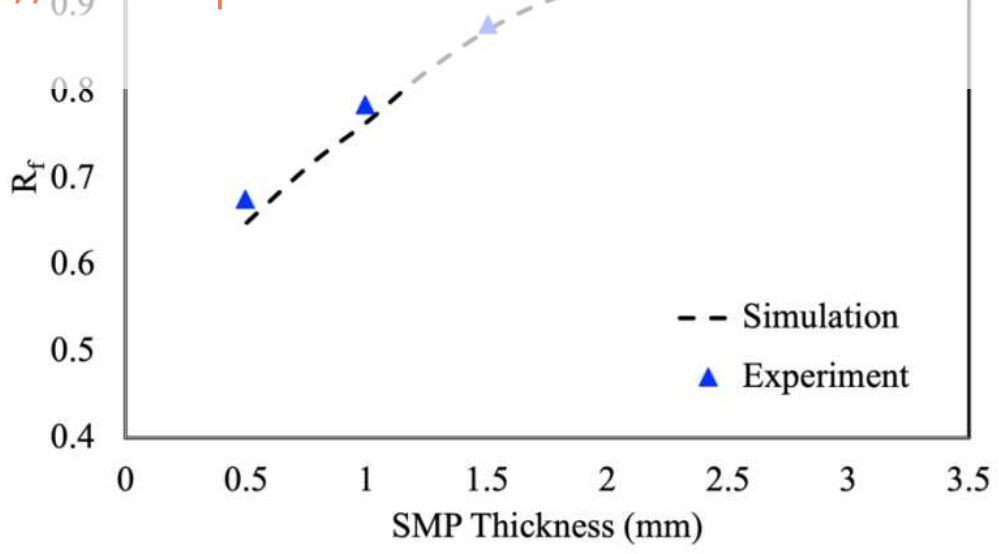

Figure 5: Variation of shape fixity ratio $\left(R_{f}\right)$ when the thickness of SMP layer is changing from $0.5 \mathrm{~mm}$ to 3 $\mathrm{mm}$.

Finally, as can be seen in Figure 6, the recovery ratio improves by increasing the thickness of the SMP layer. However, we could never reach the $100 \%$ recovery for this design of the 
SMA-SMP actuator. In general, the deposition of more SMP material in the SMA-SMP composite would increase the ability to retain the deformed shape as well as the ability to recover to the original shape. Furthermore, the proven accuracy of the finite element simulations in Figures 4 to 6 allows us to use it as part of design for manufacturing scenario.

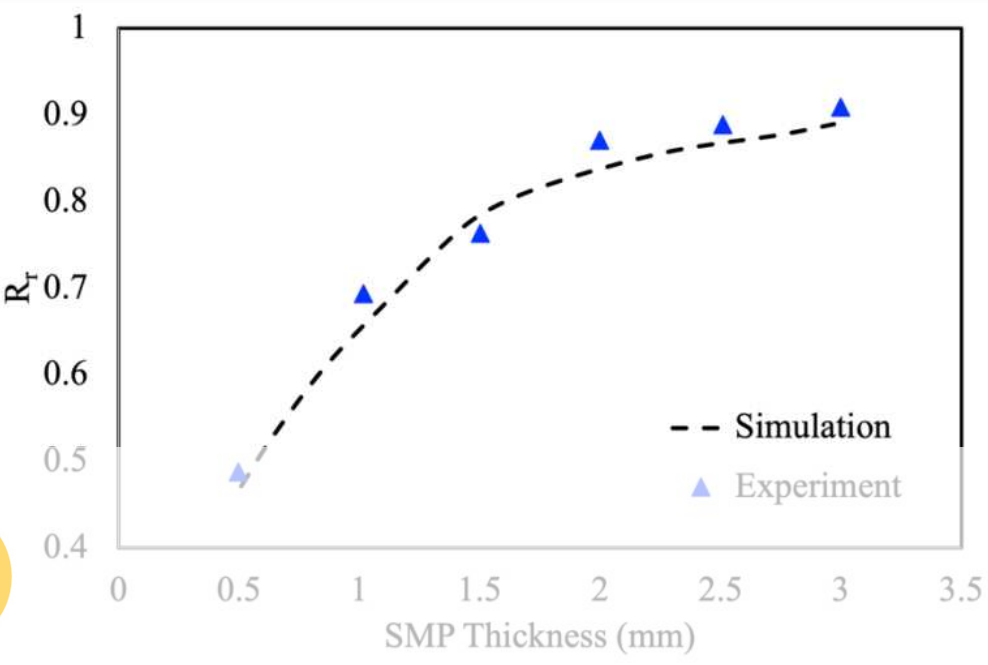

Figure 6: Variation of shape recovery ratio $\left(R_{r}\right)$ when the thickness of SMP layer is changing from $0.5 \mathrm{~mm}$ to 3
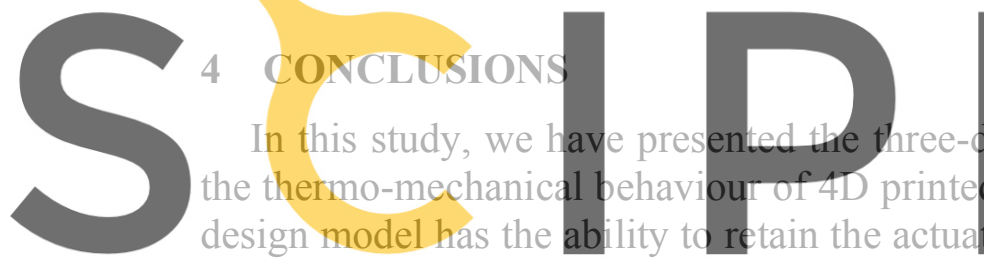
$\mathrm{mm}$.

original configuration. This is due to the implementation of the SMA wires in the SMP-

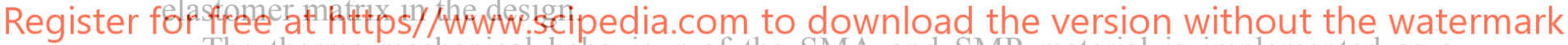

The thermo-mechanical behaviour of the SMA and SMP material is implemented as a user-defined material (UMAT) for ABAQUS from the original constitutive models that have been developed in [14-17]. Then various finite element simulations are performed by varying the thickness of the SMP segment in the SMA-SMP composite and the performance of the actuator observed.

The comparison between measurement of deformed angle $\left(\theta_{2}\right)$, shape fixity ratio $\left(R_{f}\right)$, and shape recovery ratio $\left(R_{r}\right)$ from finite element simulations and experiments validates the accuracy of the finite element models. Therefore, the proposed finite element framework could be used as part of design for manufacturing process by giving accurate prediction of the behaviour of the design. Furthermore, these measurement shows that the shape fixity and shape recovery ratio, which are important design parameters for actuator design, could be improved by increasing the thickness of the SMP layer in the model.

\section{REFERENCES}

[1] Ashir, M., Nocke, A., Theiss, C. and Cherif, C. Development of adaptive hinged fiber reinforced plastics based on shape memory alloys. Composite Structures (2017) 170:243249. 
[2] Akbari, S., Sakhaei, A.H., Panjwani, S., Kowsari, K., Serjouei, A. and Ge, Q. Multimaterial 3D printed soft actuators powered by shape memory alloy wires. Sensors and Actuators A: Physical (2019) 290:177-189.

[3] Wang, Z., Hang, G., Wang, Y., Li, J. and Du, W. Embedded SMA wire actuated biomimetic fin: a module for biomimetic underwater propulsion. Smart Materials and Structures (2008) 17(2):025039.

[4] Holschuh, B., Obropta, E. and Newman, D. Low spring index NiTi coil actuators for use in active compression garments. IEEE/ASME Transactions on Mechatronics (2014) 20(3):1264-1277.

[5] Sakhaei, A.H. and Lim, K.M. Transformation-induced plasticity in high-temperature shape memory alloys: a one-dimensional continuum model. Continuum Mechanics and Thermodynamics (2016) 28(4):1039-1047.

[6] Rodrigue, H., Wang, W., Han, M.W., Kim, T.J. and Ahn, S.H. An overview of shape memory alloy-coupled actuators and robots. Soft robotics (2017) 4(1):3-15

[7] Akbari, S. Sakhaei, A.H. Panjwani, S. Kowsari, K. and Ge, Q. Shape Memory Alloy Based 3D Printed Composite Actuators with Variable Stiffness and Large Reversible Deformation. Sensors and Actuators: A. Physical (2021) doi: https://doi.org/10.1016/j.sna.2021.112598

[8] Song, S.H., Lee, J.Y., Rodrigue, H., Choi, I.S., Kang, Y.J. and Ahn, S.H. 35 Hz shape memory alloy actuator with bending-twisting mode. Scientific reports (2016) 6(1):1-13.

[9] Wang, W., Rodrigue, H. and Ahn, S.H. Smant soft composite actuator with shape retention capability using embedded fusible alloy structures. Composites Rart B: Engineering
(2015) 78:507-514.
] Ghosh, P., Rao, A. and Srinivasa, A.R. Design of multi-state and sinart-bias components
(2013) 44:164-171.

[11] Mao, Y., Ding, Z., Yuan, C., Ai, S., Isakov, M., Wu, J., Wang, T., Dunn, M.L. and Qi,

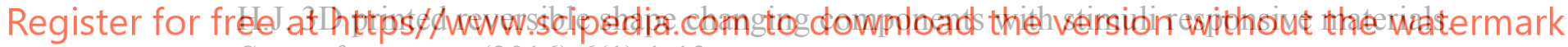
Scientific reports (2016) 6(1):1-13.

[12] Akbari, S., Sakhaei, A.H., Kowsari, K., Yang, B., Serjouei, A., Yuanfang, Z. and Ge, Q. Enhanced multimaterial 4D printing with active hinges. Smart Materials and Structures (2018) 27(6):065027.

[13] Abaqus 6.14 Documentation. Dassault Systemes Simulia Corporation, (2014).

[14] Sakhaei, A.H. and Thamburaja, P. A finite-deformation-based constitutive model for high-temperature shape-memory alloys. Mechanics of Materials (2017) 109:114-134.

[15] Lagoudas, D.C., Bo, Z. and Qidwai, M.A. A unified thermodynamic constitutive model for SMA and finite element analysis of active metal matrix composites. Mechanics of composite materials and structures (1996) 3(2):153-179.

[16] Westbrook, K.K., Kao, P.H., Castro, F., Ding, Y. and Qi, H.J. A 3D finite deformation constitutive model for amorphous shape memory polymers: a multi-branch modeling approach for nonequilibrium relaxation processes. Mechanics of Materials (2011) 43(12):853-869.

[17] Yu, K., Ge, Q. and Qi, H.J. Reduced time as a unified parameter determining fixity and free recovery of shape memory polymers. Nature communications (2014) 5(1):1-9.

[18] Arruda, E.M., Boyce, M.C. A 3-Dimensional constitutive model for the large stretch 
behavior of rubber elastic-materials. Journal of the Mechanics and Physics of Solids (1993) 41, 389-412.

[19] Sakhaei, A.H., Kaijima, S., Lee, T.L., Tan, Y.Y. and Dunn, M.L. Design and investigation of a multi-material compliant ratchet-like mechanism. Mechanism and Machine Theory (2018) 121:184-197. 